\title{
On Constrained Covariance Extension Problems
}

\author{
Kaushik Mahata and Minyue Fu
}

\begin{abstract}
This paper aims at generalizing the well-known covariance extension problem by considering additional constraints. We first consider degree constraints, i.e., we require the interpolation function to have a given degree. Several results are offered for testing the feasibility via linear matrix inequalities. We then study the spectral zero assignment problem where the the interpolation function is constrained to have the zeros of the spectral factorization of the interpolation function at given locations. A fast iterative algorithm is provided for this problem. Numerical studies support that this algorithm works extremely well, although we are yet to offer a theoretical proof for the convergence of the algorithm.
\end{abstract}

\section{INTRODUCTION}

This is a companion paper of [1] which deals with generalizations of the Nevanlinna-Pick interpolation problem by considering additional constraints. In this paper, we are concerned with similar generalizations but for the covariance extension problem. It is well known that the two problems are closely related and that most results for one problem have parallel results for the other. However, the presentations of the results and the technical tools used for developing these results may often be quite different. Therefore, it is essential that both problems are studied separately.

The classical covariance extension problem is stated as follows: Given a set of real numbers $\left\{c_{k}\right\}_{k=0}^{m}$ construct, if possible, a rational function $f(z)$ with a Laurant series expansion

$$
f(z)=\sum_{k=0}^{\infty} f_{k} z^{-k}
$$

such that

$$
f_{0}=c_{0} / 2, \quad f_{k}=c_{k}, k=0,1, \ldots, m,
$$

and that $f(z)$ is strictly positive real (SPR), i.e., $f(z)$ is analytic in $|z| \leq 1$ and that

$$
f(z)+f\left(z^{-1}\right)>0, \quad \forall|z|=1 .
$$

Like the Nevanlinna-Pick interpolation problem, the covariance extension problem has deep roots in the mathematical literature [2] and have numerous applications in various engineering fields, ranging from systems theory [3], [4] to control design [5] and signal processing [6].

In most applications, it is important that the interpolating function satisfies additional constraints. In this paper, we

This work is supported by Australian research council

The authors are with the Centre for Complex Dynamic Systems and Control, School of Electrical Engineering and Computer Science, University of Newcastle, Callaghan, NSW 2308, Australia. Minyue.Fu@newcastle.edu.au, Kaushik. Mahata@newcastle.edu.au consider two important constraints. The first one is a degree constraint, i.e., $f(z)$ is required to be of a given degree $n<m$. The second constraint is to assign the spectral zeros of $f(z)$. More precisely, let $\phi(z)$ be the unique spectral factorization of $f(z)$, i.e., $\phi(z)$ is stable with minimum phase and satisfying

$$
f(z)+f\left(z^{-1}\right)=\phi(z) \phi\left(z^{-1}\right)
$$

Then, the zeros of $\phi(z)$, which are referred to the spectral zeros of $f(z)$, need to be assigned at given locations. This interpolation problem is of interest in spectrum analysis applications [7]. This is also of interest if one wants to parameterize all the solutions via spectral zeros.

In this paper, a number of new results are presented for the aforementioned generalization problems using a new approach. For the degree constraint problem, we first establish necessary and sufficient conditions for testing whether a solution to the covariance extension problem exists for $m=2 n$ and $m=n$. This is followed by a relaxation scheme which gives a sufficient condition for testing the existence of a solution with any given $n<m<2 n$. All the tests are based on linear matrix inequalities (LMIs) and are thus computationally tractable. For the spectral zero assignment problem, we propose a new iterative algorithm which is substantially faster than the iterative algorithm in [8]. In fact, the algorithm in [8] requires solving an algebraic Riccati equation and searching an optimal Newton step size in each iteration, whereas our algorithm amounts to solving a Lyapunov equation plus some minor matrix computations in each iteration. We have tested this algorithm extensively in simulations. However, we are yet to establish theoretically that our algorithm always converges to the correct solution.

\section{Degree-CONSTRAINED COVARIANCE EXTENSION PROBLEM}

In this section, we consider the degree-constrained covariance extension problem for different values of $n$.

It is well known that the covariance extension problem without degree constraints is solvable if and only if an associated Pick matrix is positive definite [2]. If this condition holds, there always exists a solution of degree $n=m$. Another case of special interest is $m=2 n$. In this case, the solution to $f(z)$, if exists, is generically unique because $f(z)$ has $2 n+1$ coefficients and there are $m+1$ constraints in (1). We will give a necessary and sufficient condition for solving the covariance extension problem. We will also study the intermediate situation when $n<m<2 n$. A relaxation scheme is used to give a linear matrix inequality for testing if a degree-constrained solution exists or not. 
We start by introducing a simple characterization of all possible rational functions which satisfy the first $m+1$ constraints in (1), but not necessarily the SPR condition. This will help simplify calculations to a significant extent.

Lemma 1: Define the $n \times n$ matrix $F_{0}$, and the $n \times 1$ vectors $a, b, c$ and $h$ as

$$
\begin{aligned}
& F_{0}=\left[\begin{array}{cccc}
0 & 1 & \cdots & 0 \\
\vdots & \vdots & \ddots & \vdots \\
0 & 0 & \cdots & 1 \\
0 & 0 & \cdots & 0
\end{array}\right], b=\left[\begin{array}{c}
0 \\
\vdots \\
0 \\
1
\end{array}\right], h=\left[\begin{array}{c}
1 \\
0 \\
\vdots \\
0
\end{array}\right], \\
& a=\left[\begin{array}{llll}
a_{n} & a_{n-1} & \cdots & a_{1}
\end{array}\right]^{\top}, \\
& c=\left[\begin{array}{llll}
c_{1} & c_{2} & \cdots & c_{n}
\end{array}\right]^{\top} .
\end{aligned}
$$

Then the degree- $n$ rational function

$$
f(z)=c_{0} / 2+h^{\top}\left[z I-F_{0}+b a^{\top}\right]^{-1} c
$$

satisfies the interpolation conditions given by (1) upto lag $n$ for any value of $a$. Furthermore, the denominator of $f(z)$ is given by

$$
A(z)=z^{n}+a_{1} z^{n-1}+\cdots+a_{n} .
$$

Proof: The result is well known, see for example [9].

Using the lemma above we can parameterize all possible degree- $n$ rational functions satisfying (1) via the free parameter vector $a$. As $a$ determines the poles of the representation, we see that for any choice of the poles of the rational representation there exists a unique $a$ and thus a unique $f(z)$ such that (1) is satisfied. However, not every $a$ makes $f(z)$ satisfy the SPR condition (2).

\section{A. The case $m=n$}

In this case, the remaining $n$ degrees of freedom can be used to satisfy additional constraints. In [4], [8], [10], it was shown that it is possible to specify $n$ spectral zeros, provided that they are inside the closed unit disc. Therefore, the solution set is completely parameterized via spectral zeros. In what follows next, we seek for an alternative parameterization of the solution set when $m=n$.

Lemma 2: The rational function $f(z)$ in (4) is SPR real if and only if there exist a $n \times n$ positive definite matrix $Q$ and a $n \times 1$ vector $a_{\star}$ such that the following LMI (linear in terms of $Q^{-1}$ and $a^{\star}$ ) holds:

$$
\left[\begin{array}{ccc}
Q^{-1} & Q^{-1} h & Q^{-1} F_{0}^{\top}-a_{\star} b^{\top} \\
h^{\top} Q^{-1} & c_{0} & c^{\top} \\
F_{0} Q^{-1}-b a_{\star}^{\top} & c & Q^{-1}
\end{array}\right]>0
$$

If so, the vector $a$ is given by

$$
a=Q a_{\star} .
$$

Proof: Let us denote

$$
F=F_{0}-b a^{\top} .
$$

According to the Kalman-Yakubovitch-Popov lemma [11], [12], $f(z)$ in (4) is SPR if and only if there exists a positive definite matrix $Q$ such that

$$
\left[\begin{array}{cc}
Q & h \\
h^{\top} & c_{0}
\end{array}\right]-\left[\begin{array}{c}
F^{\top} \\
c^{\top}
\end{array}\right] Q\left[\begin{array}{ll}
F & c
\end{array}\right]>0
$$

Now using Schur complements this is equivalent to

$$
\left[\begin{array}{ccc}
Q & h & F^{\top} \\
h^{\top} & c_{0} & c^{\top} \\
F & c & Q^{-1}
\end{array}\right]>0
$$

Multiplying the first row of (8) by $Q^{-1}$ from the left, and the first column of (8) by $Q^{-1}$ from the right, we obtain (5).

Lemma 2 provides us with an alternative approach to parameterizing all the solutions of the rational covariance extension problem via the LMI (5) in $Q^{-1}$ and $a_{\star}$. Each feasible solution $\left(Q, a_{\star}\right)$ then gives us a feasible $a$. However it is interesting to investigate the conditions on the given data for the solvability of (5). This is established in the next Theorem.

Theorem 1: Let us define

$$
\Psi=\left[\begin{array}{cccc}
c_{0} & c_{1} & \cdots & c_{n-1} \\
c_{1} & 0 & \cdots & 0 \\
\vdots & \vdots & \ddots & \vdots \\
c_{n-1} & 0 & \cdots & 0
\end{array}\right]
$$

Then there exist $a_{\star}$ and $Q$ (Hermitian and positive definite) such that (5) holds if and only if the following LMIs

$$
Q^{-1}-F_{0}^{\top} Q^{-1} F_{0}<\Psi,\left[\begin{array}{cc}
c_{0} & c^{\prime} \\
c & Q^{-1}
\end{array}\right]>0,
$$

hold for some $Q$.

Proof: The LMI in (5) can be written as

$$
R+U a_{\star} V^{\top}+V a_{\star} U^{\top}>0,
$$

where

$$
\begin{gathered}
R=\left[\begin{array}{ccc}
Q^{-1} & Q^{-1} h & Q^{-1} F_{0}^{\top} \\
h^{\top} Q^{-1} & c_{0} & c^{\top} \\
F_{0} Q^{-1} & c & Q^{-1}
\end{array}\right], \\
U=\left[\begin{array}{cc}
I_{n} \\
0_{(n+1) \times n}
\end{array}\right], \quad V=\left[\begin{array}{c}
0_{2 n \times 1} \\
-1
\end{array}\right] .
\end{gathered}
$$

Now it is straightforward to construct the full-rank matrices $U_{\perp}$ and $V_{\perp}$ orthogonal to $U$ and $V$, respectively:

$$
U_{\perp}=\left[\begin{array}{c}
0_{n \times(n+1)} \\
I_{n+1}
\end{array}\right], V_{\perp}=\left[\begin{array}{c}
I_{2 n} \\
0_{1 \times 2 n}
\end{array}\right] .
$$

Now by the elimination lemma [12], [13] there exists $a_{\star}$ such that (5) holds if and only if $U_{\perp}^{\top} R U_{\perp}>0$ and $V_{\perp}^{\top} R V_{\perp}>0$. The first condition gives the second inequality in (9). From the second condition we get

$$
\left[\begin{array}{cc}
Q^{-1} & Q^{-1} \\
Q^{-1} & \Psi+F_{0}^{\top} Q^{-1} F_{0}
\end{array}\right]>0
$$

Applying Schur complement to (10) we get the first (Lyapunov) inequality in (9). 
We now study the conditions for (9). It is natural to expect that this condition is related to the Pick matrix associated with the interpolation data. The Pick matrix $P$ is defined as

$$
P=\left[\begin{array}{cccc}
c_{0} & c_{1} & \cdots & c_{n} \\
c_{1} & c_{0} & \cdots & c_{n-1} \\
\vdots & \vdots & \ddots & \vdots \\
c_{n} & c_{n-1} & \cdots & c_{0}
\end{array}\right]
$$

The expected result is given below.

Corollary 2: The LMI conditions given by (9) are equivalent to $P$ being positive definite.

Proof: Let $\bar{P}$ be $P$ without the last row and column. Then it is easy to verify that

$$
\bar{P}=F_{0}^{\top} \bar{P} F_{0}+\Psi, \quad P=\left[\begin{array}{cc}
c_{0} & c^{\top} \\
c & \bar{P}
\end{array}\right] .
$$

First we show the sufficiency. Suppose that $P$ is positive definite. This implies $\bar{P}$ is positive definite. Now set $Q^{-1}=$ $\bar{P}$ in (9). Comparing with (12) we see that the first inequality in (9) becomes an equality if we have $Q^{-1}=\bar{P}$, while the second inequality is satisfied. Therefore, it is possible to find $Q>0$ (by perturbing from $\bar{P}$ a little) such that both the inequalities in (9) hold.

Conversely, suppose that there exists $Q>0$ such that the LMIs in (9) hold. Then using the first equality in (12) in the first inequality in (9) we get

$$
F_{0}^{\top}\left(\bar{P}-Q^{-1}\right) F_{0}>\bar{P}-Q^{-1} \Rightarrow \bar{P}>Q^{-1}
$$

since $F_{0}$ has all eigenvalues inside the closed unit disc. Now we use the second equality in (12) and the second inequality of (9). We get

$$
P \geq\left[\begin{array}{cc}
c_{0} & c^{\top} \\
c & Q^{-1}
\end{array}\right]>0
$$

which follows from (13), and the result is proven.

\section{B. The case $m>n$}

Suppose that $f(z)$ in (4) satisfies the interpolation constraints in (1). Using the standard formula for the Laurant series expansion of $f(z)$ in (4) we get

$$
f_{0}=c_{0} / 2, \quad f_{k}=h^{\top} F^{k-1} c .
$$

Let $O=\left[\begin{array}{lllll}h & F^{\top} h & \cdots & \left(F^{\top}\right)^{n-1} h\end{array}\right]^{\top}$ be the observability matrix. The realization in (4) gives $O=I_{n}$. Consequently, we have

$$
\left[\begin{array}{lll}
c_{k} & \cdots & c_{k+n-1}
\end{array}\right]^{\top}=O F^{k-1} c=F^{k-1} c, \quad \forall k>0 .
$$

Therefore, it is straightforward that

$$
\left[\begin{array}{c}
c_{k+1} \\
\vdots \\
c_{k+n}
\end{array}\right]=F^{k} c=F\left[\begin{array}{c}
c_{k} \\
\vdots \\
c_{k+n-1}
\end{array}\right], \quad \forall k>0 .
$$

The last row of the above veector valued equality gives

$$
c_{k+n}=-\left[\begin{array}{lll}
c_{k} & \cdots & c_{k+n-1}
\end{array}\right] a, \quad \forall k>0,
$$

where we have used (6). If the number of interpolation constraints $m>n$, we get $m-n$ additional known linear constraints on $a$ in the form

$$
\bar{c}=-C a \text {. }
$$

where $C$ is a $(m-n) \times n$ Hankel matrix and $\bar{c}$ is a $(m-n) \times 1$ vector given by

$$
C=\left[\begin{array}{ccc}
c_{1} & \cdots & c_{n} \\
\vdots & \ddots & \vdots \\
c_{m-n} & \cdots & c_{m-1}
\end{array}\right], \bar{c}=\left[\begin{array}{c}
c_{n+1} \\
\vdots \\
c_{m}
\end{array}\right] .
$$

In the rest of this section, we assume that $C$ has a full row rank without any loss of generality. If $C$ does not have a full row rank, then the order of $f(z)$ must be less than $n$.

The general solution for $a$ can then be written as

$$
a=a_{0}+C_{\perp} \alpha, a_{0}=-C^{\top}\left[C C^{\top}\right]^{-1} \bar{c},
$$

where $C_{\perp}$ is a full column rank matrix of size $n \times(2 n-$ $m)$ such that $C C_{\perp}=0_{(m-n) \times(2 n-m)}$, and $\alpha$ is a $(2 n-$ $m) \times 1$ free parameter vector representing $2 n-m$ degrees of freedom.

The case $m=2 n$ is of special interest particularly in spectrum estimation applications. Here it is natural to estimate $\left\{c_{k}\right\}_{k=0}^{2 n}$ from the observed data. Subsequently a rational function is used to fit the interpolation points. When $m=2 n, C$ is a nonsingular square matrix, and there is no degree of freedom left. The solution to the interpolation problem is unique where $a=-C^{-1} \bar{c}$. Using the standard results in state space realization it is straightforward show that the minimal degree of $f(z)$ is the rank of $C$. Since $F$ is completely known for the case $m=2 n$, (7) gives a necessary and sufficient condition for $f(z)$. This is formally stated below.

Theorem 3: Suppose $m=2 n$ and $C$ in (15) is invertible. Then the interpolation problem has a unique solution given by (4) with $a=-C^{-1} \bar{c}$. This solution is SPR if and only if there exists a symmetric and positive definite $Q$ to satisfy the following LMI:

$$
\left[\begin{array}{cc}
C Q C & C h \\
h^{\top} C & c_{0}
\end{array}\right]>\left[\begin{array}{c}
C F_{0}^{\top}+\bar{c} b^{\top} \\
c^{\top}
\end{array}\right] Q\left[\begin{array}{ll}
F_{0} C+b \bar{c}^{\top} & c
\end{array}\right] .
$$

Proof: The solution to a follows from the discussion prior to the theorem and the LMI condition follows from Lemma 2 (see (7) in particular).

Next we investigate the case when $n<m<2 n$. Naturally, in this case the solvability conditions become more stringent as we increase $m$. In the following lemma we formulate the solvability conditions.

Lemma 3: Assume that $n<m<2 n$. Then there exists $\alpha$ as in (16) such that $f(z)$ in (4) is SPR if and only if

$$
Q^{-1}-F_{0}^{\top} Q^{-1} F_{0}<\Psi
$$

and

$$
\left[\begin{array}{cc}
C Q C^{\top} & C h \\
h^{\top} C^{\top} & c_{0}
\end{array}\right]>\left[\begin{array}{c}
C F_{0}^{\top}+\bar{c} b^{\top} \\
c^{\top}
\end{array}\right] Q\left[\begin{array}{c}
C F_{0}^{\top}+\bar{c} b^{\top} \\
c^{\top}
\end{array}\right]_{(18)}^{\top}
$$


for some symmetric and positive definite $Q$.

Proof: First we modify the SPR condition in (8) by using (16) to get

$$
\left[\begin{array}{ccc}
Q & h & F_{0}^{\top}-b a_{0}^{\top} \\
h^{\top} & c_{0} & c^{\top} \\
F_{0}-a_{0} b^{\top} & c & Q^{-1}
\end{array}\right]+V \alpha^{\top} \bar{U}^{\top}+\bar{U} \alpha V>0,
$$

where $V$ is given as in the proof of Theorem 1 and

$$
\bar{U}=\left[\begin{array}{c}
C_{\perp} \\
0_{(n+1) \times(2 n-m)}
\end{array}\right] .
$$

It is readily verified that the columns of

$$
\bar{U}_{\perp}=\left[\begin{array}{cc}
C & 0_{(m-n) \times(2 n+1)} \\
0_{(2 n+1) \times(m-n)} & I_{2 n+1}
\end{array}\right]
$$

spans the orthogonal complement of the row-space of $\bar{U}$. Therefore by the elimination lemma, there exist $\alpha$ and $Q$ such that (19) is satisfied if and only if (10) holds along with

$$
\left[\begin{array}{ccc}
C Q C^{\top} & C h & C\left(F_{0}^{\top}-a_{0} b^{\top}\right) \\
h^{\top} C^{\top} & c_{0} & c^{\top} \\
\left(F_{0}-b a_{0}^{\top}\right) C^{\top} & c & Q^{-1}
\end{array}\right]>0 .
$$

The inequality (17) follows from (10) as before and (18) follows by applying Schur complement to (20).

It is interesting to see how the additional interpolation constraints impose more and more stringent requirements for solvability of the problem. Comparing (20) with the second inequality in (9) we see that when $m=n$ we only need to satisfy the inequality obtained by taking the last $n+1$ rows and last $n+1$ columns of (20). The $(n+k)$-th interpolation condition then adds the $k$-th row and the $k$-th column in (20). It is straightforward to see that the solvabiliy for $n+$ $k$ interpolation constraints implies solvability for $n+k-$ 1 interpolation conditions, but converse is not true. Finally, when $m=2 n$, the feasible set contain a maximum of one point.

It is evident that Lemma 3 gives a non-convex set in $Q$, and it is in general difficult to solve the LMIs in Lemma 3 using any numerical technique. For this purpose we propose a relaxation scheme in the following. The idea of the relaxation scheme is to find a convex subset of the set of feasible $Q$ given by Lemma 3 .

Theorem 4: Consider the case when $n<m<2 n$. Then there exists a solution to the interpolation problem if the following LMI (linear in $Q^{-1}$ and

$$
\begin{aligned}
{\left[\begin{array}{cc}
c_{0} & c^{\top} \\
c & Q^{-1}
\end{array}\right]>} & {\left[\begin{array}{c}
h^{\top} \Pi_{C} \\
F_{0}^{\top} \Pi_{C}+b \bar{c}^{\top} C^{\dagger \top}
\end{array}\right] } \\
& \cdot Q^{-1}\left[\begin{array}{c}
h^{\top} \Pi_{C} \\
F_{0}^{\top} \Pi_{C}+b \bar{c}^{\top} C^{\dagger \top}
\end{array}\right]
\end{aligned}
$$

is feasible in a positive definite matrix $Q$, where

$$
C^{\dagger}=C^{*}\left(C C^{*}\right)^{-1}, \quad \Pi_{C}=C^{\dagger} C .
$$

Proof: First note that feasibility of the LMI

$$
\left[\begin{array}{cccc}
C_{\perp}^{\top} Q C_{\perp} & C_{\perp}^{\top} Q C^{\top} & 0 & 0 \\
C Q C_{\perp} & C Q C^{\top} & C h & C\left(F_{0}^{\top}-a_{0} b^{\top}\right) \\
0 & h^{\top} C^{\top} & c_{0} & c^{\top} \\
0 & \left(F_{0}-b a_{0}^{\top}\right) C^{\top} & c & Q^{-1}
\end{array}\right]>0
$$

in $Q$ implies (20). Now taking the Schur complement of (22) we have

$$
\begin{aligned}
& {\left[\begin{array}{cc}
c_{0} & c^{\top} \\
c & Q^{-1}
\end{array}\right]>\left[\begin{array}{cc}
0 & h^{\top} C^{\top} \\
0 & \left(F_{0}-b a_{0}^{\top}\right) C^{\top}
\end{array}\right] \times} \\
& {\left[\begin{array}{ll}
C_{\perp} & C^{\top}
\end{array}\right]^{-1} Q^{-1}\left[\begin{array}{c}
C_{\perp}^{\top} \\
C
\end{array}\right]^{-1}\left[\begin{array}{cc}
0 & h^{\top} C^{\top} \\
0 & \left(F_{0}-b a_{0}^{\top}\right) C^{\top}
\end{array}\right]^{\top}(23)}
\end{aligned}
$$

Now note that

$$
\left[\begin{array}{c}
C_{\perp}^{\top} \\
C
\end{array}\right]^{-1}=\left[\begin{array}{ll}
C_{\perp}^{\dagger \top} & C^{\dagger}
\end{array}\right]
$$

which we substitute in (23) to get (21).

We point out that even if the LMI (21) is not feasible in $Q$, the interpolation problem may still have a solution.

\section{SPECTRAL ZERO ASSIGNMENT}

In this section we reconsider the case $m=n$. Here our objective is to derive a fast algorithm for the covariance extension problem subject to spectral zero assignment. To this end, we first recite a key result in [14], [15].

Theorem 5: Let the Pick matrix $P$ in (11) be positive definite and $n=m$. Then for every monic marginally Schur stable polynomial $\bar{\sigma}(z)$ of order $n$, there exists a unique $n$-th order monic Schur stable polynomial $\bar{\tau}(z)$ such that

$$
f(z)+f^{*}\left(z^{-1}\right)=\rho^{2} \frac{\bar{\sigma}^{*}\left(z^{-1}\right)}{\bar{\tau}^{*}\left(z^{-1}\right)} \frac{\bar{\sigma}(z)}{\bar{\tau}(z)}
$$

such that the interpolation conditions in (1) are satisfied, where the gain $\rho$ is determined by $f(\infty)=f_{0}=c_{0} / 2$.

Proof: The result above comes from [4], [8], [10].

In contrast to the previous section we take a different approach here. In the previous section we formulated a state space realization of $f(z)$ such that the interpolation conditions in (1) are automatically satisfied. Here we parameterize the minimum phase spectral factor $\bar{\sigma}(z) / \bar{\tau}(z)$ of $f(z)+f^{*}\left(z^{-1}\right)$ such that the spectral zeros are automatically assigned to the desired points. Now the free parameters are the coefficients of denominator polynomial $\bar{\tau}(z)$. Hence it remains to tune only the coefficients of $\bar{\tau}(z)$ so that the interpolation conditions in (1) are satisfied while maintaining the SPR condition.

We express

$$
\begin{aligned}
\bar{\sigma}(z) & =z^{n}+\sigma_{1} z^{n-1}+\cdots+\sigma_{n} \\
\bar{\tau}(z) & =z^{n}+\tau_{1} z^{n-1}+\cdots+\tau_{n} .
\end{aligned}
$$

and denote

$$
\sigma=\left[\begin{array}{lll}
\sigma_{1} & \cdots & \sigma_{n},
\end{array}\right]^{\top} ; \tau=\left[\begin{array}{lll}
\tau_{1} & \cdots & \tau_{n}
\end{array}\right]^{\top} .
$$


Let us introduce the matrix

$$
A=F_{0}-\tau h^{\top} .
$$

Hence the problem under consideration is to find $\tau$ such that the interpolation conditions in (1) are satisfied. Using the observable canonical state-space realization, we have

$$
\frac{\bar{\sigma}(z)}{\bar{\tau}(z)}=1+h^{\top}\left[z I_{n}-A\right]^{-1}(\sigma-\tau) .
$$

Then $f(z)$ in (24) is given by [4]

$f(z)=\frac{\rho^{2}}{2}\left(1+h^{\top} R h\right)+\rho^{2} h^{\top}[z I-A]^{-1}\{A R h+(\sigma-\tau)\}$,

where $R$ satisfies

$$
R=A R A^{\top}+(\sigma-\tau)(\sigma-\tau)^{\top} .
$$

We now explore the relationship between $\tau$ and $\sigma$ given the interpolation conditions in (1).

Lemma 4: Let us define the Toeplitz matrix

$$
L=\left[\begin{array}{cccc}
c_{0} & 0 & \cdots & 0 \\
c_{1} & c_{0} & \cdots & 0 \\
\vdots & \ddots & \ddots & \vdots \\
c_{n-1} & \cdots & c_{1} & c_{0}
\end{array}\right]
$$

Then $f(z)$ in (27) satisfies the interpolation conditions in (1) if and only if

$$
\tau=\frac{c_{0}}{1+h^{\top} R h} L^{-1}\left(F_{0} R h+\sigma\right)-L^{-1} c,
$$

and

$$
\rho^{2}=\frac{c_{0}}{1+h^{\top} R h} .
$$

where $R$ satisfies (28).

Proof: Taking $z \rightarrow \infty$ in (27) we get

$$
f(\infty)=\frac{\rho^{2}}{2}\left(1+h^{\top} R h\right) .
$$

Now imposing the the interpolation condition at $z=\infty$ we get (30). Now expanding $f(z)$ in (27) in Laurant series and using the remaining interpolation conditions we get

$$
c=\rho^{2} O\{A R h+(\sigma-\tau)\} .
$$

It can be verified that

$$
O^{-1}=I_{n}+\tau_{1} F_{0}^{\top}+\tau_{2}\left(F_{0}^{\top}\right)^{2}+\cdots+\tau_{n-1}\left(F_{0}^{\top}\right)^{n-1} .
$$

Also from (25) and (30) we see that

$$
\rho^{2}\{A R h+(\sigma-\tau)\}=\rho^{2}\left(F_{0} R h+\sigma\right)-c_{0} \tau .
$$

Combining (32), (33) and (34) we get

$$
L \tau+c=\rho^{2}\left(F_{0} R h+\sigma\right),
$$

which leads us to (29) using (30).

Lemma 4 gives us a way to find $\tau$. Note that (28) is a generalized Riccati equation because (29) gives $\tau$ as a function of $R$. Thus, solving (28) directly may be difficult. Instead we can solve $R$ using (28) and (29) repeatedly until convergence. The Recursion is as follows. Initialize $k=0$ and $R_{0}=I_{n}$. Then the following steps are repeated until convergence:

1) $\rho_{k}^{2}=\frac{c_{0}}{1+h^{\top} R_{k} h}$,

2) $\tau^{(k)}=\rho_{k}^{2} L^{-1}\left(F_{0} R_{k} h+\sigma\right)-L^{-1} c$,

3) $A_{k}=F_{0}-\tau^{(k)} h^{\top}$

4) $R_{k+1}=A_{k} R_{k} A_{k}^{\top}+\left(\sigma-\tau^{(k)}\right)\left(\sigma-\tau^{(k)}\right)^{\top}$

Although we do not have a theoretical justification that the recursion described above converges, extensive numerical study has shown that the recursion does always converge. It is also interesting to compare the above recursive algorithm with the Byrnes-Georgiou-Lindquist algorithm proposed in [4]. In [4], a generalized entropy functional is maximized and the underlying optimization problem is shown to be convex. The convex function is then minimized using a gradient decent method. However, each iteration of the gradient decent amounts to solving a Riccati equation. The proposed algorithm involves solving a Lyapunov equation (Step 4) plus some minor computations (Steps 1, 2 and 3). This makes the proposed algorithm much more efficient from a practical point of view.

\section{CONCLUSION}

We have provided a number of new results on constrained covariance extension problems. The results on degree constrained problems are derived based on a new parameterization of the unconstrained solution set, as given in Lemmas 1 and 2. This leads to a number of LMI based tests for the solvability of degree constrained interpolation problem. For the cases of $m=2 n, m=n$ and $m=n+1$, the tests are exact. For the case of $n+1<m<2 n$, it would be interesting to see whether the relaxation scheme can be further improved. For the spectral zero assignment problem, our solution is based on a totally different parameterization of the unconstrained solution set, as given in Lemma 4. The result is a very fast iterative algorithm, although its convergence is yet to be established theoretically.

\section{REFERENCES}

[1] M. Fu and K. Mahata, "Generalizations of the Nevanlinna-Pick interpolation problem," in 44th IEEE Conference on Decision and Control, Sevile, Spain, December 2005.

[2] N. I. Akhiezer, The classical moment problem. London: Oliver and Boyd, 1965.

[3] P. Delsarte, Y. Genin, and Y. Kamp, "On the role of the NevanlinnaPick problem in circuits and system theory," Circuit Theory and Applicaions, vol. 9, pp. 177-187, 1981.

[4] C. I. Byrnes and A. Lindquist, "On the partial stochastic realization problem," IEEE Transactions on Automatic Control, vol. 42:8, pp. 1049-1070, August 1997.

[5] H. Kimura, "Robust stabilizability for a class of transfer functions," IEEE Transactions on Automatic Control, vol. AC-29, pp. 788-793, September 1984.

[6] T. T. Georgiou, "Realization of power spectra from partial covariance sequences," IEEE Transactions on Acoustics, Speech and Signal Processing, vol. ASSP-35, pp. 438-449, 1987.

[7] C. I. Byrnes, T. T. Georgiou, and A. Lindquist, "A new approach to spectral estimation: A tunable high-resolution spectral estimator," IEEE Transactions on Signal Processing, vol. 48:4, pp. 3189-3205, November 2000. 
[8] C. I. Byrnes, A. Lindquist, and S. V. Gusev, "A convex optimization approach to the rational covariance extension problem," SIAM Journal of Control Optimization, vol. 37, pp. 211-229, 1999.

[9] T. Kailath, Linear Systems. Prentice Hall, 1980.

[10] C. I. Byrnes, A. Lindquist, S. V. Gusev, and A. S. Matveev, "A complete parameterization of all positive rational extensions of a covariance sequence," IEEE Transactions on Automatic Control, vol. 40:11, pp. 1841-1857, November 1995.

[11] A. Rantzer, "On the Kalman-Yakubovitz-Popov lemma," Systems \& Control Letters, vol. 28, pp. 7-10, 1996.

[12] S. Boyd, L. E. Ghaoui, E. Feron, and V. Balakrishnan, Linear Matrix Inequalities in System and Control Theory. SIAM, 1994.

[13] E. van der Meché and O. Bosgra, "Nevanlinna-Pick interpolation with degree constraints: complete characterization based on Lyapunoov inequalities," in 43rd IEEE conference on Decision and Control, Atlantis, Paradise Islands, Bahamas, December 2004, pp. 471-476.

[14] C. I. Byrnes, T. T. Georgiou, and A. Lindquist, "A generalized entropy criterion for Nevanlinna-Pick interpolation with degree constraint," IEEE Transactions on Automatic Control, vol. 46:6, pp. 822-839, June 2001.

[15] T. T. Georgiou, "The interpolation problem with a degree constraint," IEEE Transactions on Automatic Control, vol. 44:3, pp. 631-635, March 1999. 